\title{
The Jacobson Radical of the Endomorphism Semiring of P.Q.- Principal Injective Semimodules
}

\author{
Khitam S. H. Aljebory *
}

\author{
Assad M. A. Alhossaini
}

Received 12/3/2019, Accepted 21/10/2019, Published 1/6/2020

This work is licensed under a Creative Commons Attribution 4.0 International License.

\begin{abstract}
:
In this work, we introduced the Jacobson radical (shortly Rad $(S)$ ) of the endomorphism semiring $S$ $=\operatorname{End}_{\mathcal{R}}(\mathcal{B})$, provided that $\mathcal{B}$ is principal P.Q.- injective semimodule and some related concepts, we studied some properties and added conditions that we needed. The most prominent result is obtained in section three -If $\mathcal{B}$ is a principal self-generator semimodule, then $Z(S, S)=W(S$,$) .$
\end{abstract}

Subject Classification: 16y60

Key words: Jacobson radical of semiring, P-injective, P.Q.- principal injective semimodule, Semimodule semiring.

\section{Introduction:}

This paper is interested in the generalization of some results in ring theory and module theory. Semirings and semimodules and their applications; grow in different branches of mathematics, computer sciences, physics, also in many other areas of modern science. The study of semimodules over semiring has been extensively considered, as reviewed by Golan in (1) and references therein. Semirings are moved from rings but simultaneously there are important differences between them. A semiring is a nonempty set $\mathcal{R}$ together with two operations, addition and multiplication, where these two operations are associative, whereas addition is a commutative operation, the distribution law holds, there is $0 \in \mathcal{R}$ (additive identity element) such that $t+0=t=0+t, t 0=0 t=0$ for each $\mathrm{t}$ in $\mathcal{R}$ and there is multiplicative identity element (denoted 1 ) where $1 \neq 0$. It is commutative if the second operation is commutative. For instance, the set of natural number $\mathbb{N}$ is a commutative semiring under usual addition and multiplication, but it is not ring. A semimodule $\mathcal{B}$ over semiring $\mathcal{R}$ is defined similarly in module over ring(1). In 1945 Nathan Jacobson was the first to study the Jacobson radical for arbitrary rings, it is denoted by $\mathrm{J}(R)$ or $\operatorname{rad}(R)$, so it was called after his name, where he defined the

College of Education for Pure Sciences, University of Babylon, Babylon, Iraq.

*Corresponding author:khitam sahib25@gmail.com.

*ORCID ID: https://orcid.org/5568-0692-0003-0000
Jacobson radical of a ring $R$ to be the ideal consisting of those elements in $R$ that annihilate left $R$-module and then was given by $\operatorname{Kasch}(1982)(2)$, Anderson(1992), Isaacs(1993) and $\operatorname{Lam}(2001)$ with equivalent definitions. They defined $\mathrm{J}(R)$ to be the intersection of all maximal left ideals of the ring which equals the sum of all superfluous left ideals. Analogously, in this paper we study the Jacobson radical of endomorphism semiring $S$ of an $\mathcal{R}$ semimodule, in particular of P.Q.-injective semimodule (3). For $S=\operatorname{End}_{\mathcal{R}}(\mathcal{B})$, we define the Jacobson radical of $S$, socle semimodule, singular and symbolizes them respectively by $\operatorname{Rad}(S)$, $\operatorname{Soc}(\mathcal{B}), Z(S) \cdot \operatorname{Rad}(S)=\bigcap\{J: J$ is maximal left ideals of $S\}=$ the set of all non-invertible elements of $S(4) . Z(S)=,\{s \in S \mid \operatorname{ann}(s)$ is essential ideal of $S\}(5), \quad \operatorname{Soc}(\mathcal{B})=\cap\{U \mid U$ is essential subsemimodule of $\mathcal{B}\}=\sum\{L: L$ is a simple $\mathcal{R}$ subsemimodule of $\mathcal{B}\}(3)$. Also we define the concept "Kacsh semimodule" similar to what is defined in module (6), as well the concept principally self-generator semimodule and we show that, if $\mathcal{B}$ is principally self-generator with $S=$ $E n d_{\mathcal{R}}(\mathcal{B})$ we have $Z(S)=W(S)$ where $W(S)=\{$ $\omega \in S \mid \operatorname{ker} \omega$ is essential in $\mathcal{B}\}$.

We define condition for $\mathcal{B}$ called $\mathrm{C}_{2}$-condition, where an $\mathcal{R}$-semmimodule $\mathcal{B}$ is said to satisfy the $\mathrm{C}_{2}$-condition if each subsemimodule of $\mathcal{B}$ which is isomorphic to a direct summand of $\mathcal{B}$ is itself a direct summand of $\mathcal{B}$, it is shown that every cyclic P.Q.-injective semimodule has $\mathrm{C}_{2}$ - condition and, if $\mathcal{B}$ has $\mathrm{C}_{2}$ - condition then $W(S, \subseteq \operatorname{Rad}(S$,$) in module$ 
theory, but in semimodule theory we must add some conditions for $\mathcal{B}$ in order to get $W(S, \subseteq \operatorname{Rad}(S)$.

Throughout this paper all semirings are commutative with identity and all semimodules are unitary.

In the following, we review some definitions and remarks that will be applied in this paper.

\section{Preliminaries \\ Definition 1. (3). Let $\mathcal{R}$ be a semiring, then for any $a \in \mathcal{R}$, \\ $\mathcal{R} a=\{x: x=t a\}$ for some $t \in \mathcal{R}$. It is a left ideal of $\mathcal{R}$ called the principal left ideal generated by $a$.}

Definition 2. (4). The Jacobson radical of a semiring $\mathcal{R}$, denoted by $\operatorname{Rad}(\mathcal{R})$, is the intersection of all maximal left ideal of $\mathcal{R}$. For instance, $\operatorname{Rad}\left(\mathbb{Z}_{4}\right)=\{\overline{0}, \overline{2}\}$.

Definition 3. (4). A semiring $\mathcal{R}$ is called a left local semiring if it has a unique maximal left ideal.

Theorem 4. (4). Let $\mathcal{R}$ be semiring, then the following statements are equivalent:

(a) $\mathcal{R}$ is local.

(b) $\operatorname{Rad}(\mathcal{R})$ is maximal ideal of $\mathcal{R}$.

(c)The set of all non-invertible elements is an ideal of $\mathcal{R}$.

(d) $\operatorname{Rad}(\mathcal{R})=\{r \in \mathcal{R} \mid r$ is non-invertible $\}$.

Definition 5. (1). A nonempty subset $U$ of semimodule $\mathcal{B}$ is called subsemimodule if $b, b^{\prime} \in$ $\mathcal{B}, t \in \mathcal{R}$, then $b+b^{\prime}$ and $t b \in U$. It is denoted by $U$ $\leq \mathcal{B}$.

Definition 6. (7). Let $\mathcal{R}$ be a semiring and $U \leq \mathcal{B}$, then $U$ is said to be a direct summand of $\mathcal{B}$ if there exists subsemimodule $C$ of $\mathcal{B}$, such that $\mathcal{B}=U \oplus C$ and $\mathcal{B}$ is called a direct sum of $U$ and $C$.

Definition7. (1). A subsemimodule $U$ of an $\mathcal{R}$ semimodule $\mathcal{B}$ is called subtractive if $\forall b, b^{\prime} \in \mathcal{B}$ in which $b+b^{\prime}, b \in U$, then $b^{\prime} \in U$. A semimodule is subtractive if all subsemimodules of it is subtractive. It is clear that $\{0\}$ is subtractive of any semimodule.

Definition 8. (8). An element $b$ of a semimodule $\mathcal{B}$ is cancellable if $b+c=b+d$ implies that $c=d$. The semimodule $\mathcal{B}$ is cancellative if every element of $\mathcal{B}$ is cancellable.

Definition 9. (3). A semimodule $\mathcal{B}$ is called a semisubtractive, if for any $b, b^{\prime} \in \mathcal{B}$ there is always some $h \in \mathcal{B}$ satisfying $b+h=b^{\prime}$ or some $k \in \mathcal{B}$ satisfying $b^{\prime}+k=b$.

Definition 10. (5). A subsemimodule $U$ of an $\mathcal{R}$ semimodule $\mathcal{B}$ is called maximal subsemimodule of $\mathcal{B}$ if it is not contained properly in any other proper subsemimodule of $\mathcal{B}$.
Definition 11. (9). A subsemimodule $U$ of a semimodule $\mathcal{B}$ is called essential (large) subsemimodule of $\mathcal{B}$, if $U \cap C=0$ implies $C=0$ where $C$ is any subsemimodule of $\mathcal{B}$, notational $U$ $\leq_{e} \mathcal{B}$.

Definition 12. (10). A semimodule $\mathcal{B}$ is called uniform semimodule if the intersection of any nonzero two subtractive subsemimodules of $\mathcal{B}$ is nonzero subsemimodule of $\mathcal{B}$.

Definition 13. (5). Let $\mathcal{B}$ be a semimodule and $b \in \mathcal{B}$. The left annihilator of $b$ is defined by $\operatorname{ann}_{R}(b)=\{t \in \mathcal{R} \mid t b=0\}$. Obviously $\operatorname{ann}_{R}(b)$ is a left ideal of $\mathcal{R}$. If $U$ is a subsemimodule of $\mathcal{B}$, then $\operatorname{ann}_{R}(U)=\{t \in \mathcal{R} \mid t b=0, \forall b \in U\}$ is ideal of $\mathcal{R}$.

Definition 14. (5). The singular subsemimodule of $\mathcal{B}$ is defined by $Z(b)=\left\{b \in \mathcal{B} \mid a n n_{R}(b)\right.$ is essential ideal in $\mathcal{R}$. If $Z(\mathcal{B})=\mathcal{B}, \mathcal{B}$ is called singular and if $Z(\mathcal{B})=0$, then $\mathcal{B}$ is called nonsingular semimodule. The singular subsemimodule of the left semimodule ${ }_{R} \mathcal{R}$ is called the (left) singular ideal of the semiring $\mathcal{R}$ and is denoted by $Z(\mathcal{R})$, i.e. $Z(\mathcal{R})=\{t \in \mathcal{R}$ : $a_{n} n_{R}(t) \cap H \neq 0$ for every nonzero left ideal $H$ of $\left.\mathcal{R}\right\}$.

Remark. 15. (5). $Z(\mathcal{R})$ is an ideal of $\mathcal{R}$.

Definition 16. (5). A subsemimodule $S$ of $\mathcal{B}$ is called small (superfluous) if for any subsemimodule $S^{\prime}$ of $\mathcal{B}, S+S^{\prime}=\mathcal{B}$ implies $S^{\prime}=\mathcal{B}$.

Definition 17. (5). The radical of a semimodule $\mathcal{B}$ defined by $\operatorname{Rad}(\mathcal{B})=\bigcap\{\quad U: \quad U$ is maximal subsemimodule of $\mathcal{B}$ \}.

Remark 18. (5). $\operatorname{Rad}(\mathcal{B})=\{\quad U: \quad U$ is maximal subsemimodule in $\mathcal{B}\}=\sum\{S: \quad S$ is small subsemimodule in $\mathcal{B}$ \}.

Proposition 19. (5). Let $\mathcal{B}$ be left $\mathcal{R}$-semimodule, if $\mathcal{B}$ is finitely generated, then $\operatorname{Rad}(\mathcal{B})$ is superfluous subsemimodule of $\mathcal{B}$.

Definition 20. (3). Let $\mathcal{B}$ be a semimodule, the sum of all simple subsemimodules of $\mathcal{B}$ is called the socle of $\mathcal{B}$, which is equal to the intersection of all essential subsemimodules of $\mathcal{B}$, it is denoted by $\operatorname{Soc}(\mathcal{B})$. If $\mathcal{B}$ has no simple subsemimodule then we put $\operatorname{Soc}(\mathcal{B})=0$.

Definition 21.(11). A semimodule $\mathcal{B}$ is said to be semi-simple if it is a direct sum of its simple subsemimodules $\mathcal{B}$.

Remark 22. (3). If $\operatorname{Soc}(\mathcal{B})=\mathcal{B}$, then $\mathcal{B}$ is semisimple.

Definition 23. (5). Let $\mathcal{B}$ and $\mathcal{B}^{\prime}$ be two $\mathcal{R}$ semimodules. A mapping $\phi: \mathcal{B} \rightarrow \mathcal{B}^{\prime}$ is said to be a homomorphism of $\mathcal{R}$-semimodules if $\phi\left(b+b^{\prime}\right)=$ $\phi(b)+\phi\left(b^{\prime}\right)$ and $\phi(t b)=t \phi(b), \forall b, b^{\prime} \in \mathcal{B}$ and $t \in$ $\mathcal{R}$.

The set of $\mathcal{R}$-homomorphisms of $\mathcal{B}$ into $N$ is denoted by $\operatorname{Hom}(\mathcal{B}, N)$. A homomorphism $\psi$ is called an epimorphism if its onto, it is called a 
monomorphism if $\psi$ is one-one and it is isomorphism if $\psi$ is one-one and onto. Also we denoted $\operatorname{End}_{\mathcal{R}}(\mathcal{B})$ is the set $S$ of endomorphisms of $\mathcal{B}$.

Remark 24. (5). For a homomorphism $\phi: \mathcal{B} \rightarrow \mathcal{B}^{\prime}$ of left $\mathcal{R}$-semimodules we define

(a) $\operatorname{ker}(\phi)=\{b \in \mathcal{B} \mid \phi(b)=0\}$ which is a subtractive subsemimodule of $\mathcal{B}$.

(b) $\phi(\mathcal{B})=\{\phi(b) \mid b \in \mathcal{B}\}$. It is subsemimodule of $\mathcal{B}^{\prime}$.

(c) $\operatorname{Im}(\phi)=\left\{b^{\prime} \in \mathcal{B}^{\prime} \mid b^{\prime}+\phi(b)=\phi\left(b^{\prime \prime}\right)\right.$ for some $b$, $\left.b^{\prime \prime} \in \mathcal{B}\right\}$ also is a subtractive subsemimodule of $\mathcal{B}^{\prime}$. It is clear that $\phi(\mathcal{B}) \subseteq \operatorname{Im}(\phi)$. The equality is satisfied if $\phi(\mathcal{B})$ is subtractive

Definition 25.(3). An $\mathcal{R}$-semimodule $E$ is $\mathcal{B}$ injective ( $E$ is injective relative to $\mathcal{B}$ ) if, for each subsemimodule $U$ of $\mathcal{B}$, any $\mathcal{R}$-homomorphism from $U$ to $E$ can be extended to an $\mathcal{R}$ homomorphism from $\mathcal{B}$ to $E$. A left $\mathcal{R}$-semimodule $E$ is injective if it is injective relative to every left $\mathcal{R}$-semimodule. A semimodule $\mathcal{B}$ is quasi -injective if it is $\mathcal{B}$-injective.

Remark 26. Every injective semimodule is quasiinjective but the converse is not true.

For example, $\mathbb{Z} / 2 \mathbb{Z}$ as $\mathbb{N}$-semimodule is quasiinjective but it is not injective.

In (6) a generalization for injective modules were given, in (3) the following concept was given analogous to that concept for semimodules.

Definition 27.(3). An $\mathcal{R}$-semimodule is called principally quasi-injective (P.Q.-injective) if each homomorphism from cyclic (principal) subsemimodule of $\mathcal{B}$ to $\mathcal{B}$ can be extended to an endomorphism of $\mathcal{B}$.

Examples 28.

(a) Every injective semimodule is P.Q.-injective .

(b) Every semi-simple semimodule is P.Q.injective.

(c) $\mathbb{Z}_{2}$ as $\mathbb{N}$-semimodule is P.Q.-injective but not not injective.

Definition 29. (3). An $\mathcal{R}$-semimodule $\mathcal{B}$ is called P-injective if for any principal ideal $I$ of $\mathcal{R}$ and each $\mathcal{R}$-homomorphism $\alpha: I \rightarrow \mathcal{B}$, there exists an $\mathcal{R}$ homomorphism $\gamma: \mathcal{R} \rightarrow \mathcal{B}$, which extends $\alpha$.

\section{The Jacobson radical of endomorphism semiring and some related concepts}

In this part we study the Jacobson radical of endomorphism semiring, in particular for P.Q.injective semimodule and some related concepts. We add some remarks that help us to avoid some problems which we encountered. For $S=\operatorname{End}_{\mathcal{R}}(\mathcal{B})$, we discuss $\mathcal{Z}(S), \operatorname{Soc}(\mathcal{B}), W(S)$. We introduce the concept of kasch semimodule, and study their relationship with Jacobson radical, also, $\mathrm{C}_{2^{-}}$ condition, where semimodule is said to satisfy the $\mathrm{C}_{2}$-condition if every subsemimodule of $\mathcal{B}$ which is isomorphic to a direct summand of $\mathcal{B}$ is itself a direct summand of $\mathcal{B}$, and principally self generator, an $\mathcal{R}$-semimodule $\mathcal{B}$ is said to be principally self-generator if for every element $b \in \mathcal{B}$, there exists an epimorphism $\alpha: \mathcal{B} \rightarrow \mathcal{R} b$. These concepts are mentioned for modules in (6) and (12).

In this section we considered $\mathcal{B}$ is semimodule with $S=\operatorname{End}_{\mathcal{R}}(\mathcal{B})$.

The following lemma was proved in [Kasch, p.110](2) for module, similar, we prove this lemma for semimodule.

Lemma 1. If $U \leq_{e} \mathcal{B}^{\prime}$ and $\alpha \in \operatorname{Hom}\left(\mathcal{B}, \mathcal{B}^{\prime}\right)$, then $\alpha^{-1}(U) \leq_{e} \mathcal{B}$.

Proof: Let $V$ subsemimodule of $\mathcal{B}$ and $\alpha^{-1}(U) \cap V=$ 0 implies $U \cap \alpha(V)=0$ (since $U \leq_{e} \mathcal{B}^{\prime}$ ), then $\alpha(V)$ $=0 \Rightarrow V \leq \operatorname{ker}(\alpha)=\alpha^{-1}(0) \leq \alpha^{-1}(U) \Rightarrow V=$ $\alpha^{-1}(U) \cap V=0 . \quad$ /III

Remark 2.Let $\mathcal{B}$ be an $\mathcal{R}$-semimodule with $S$, then the set $W(S)=,\{\omega \in S, \operatorname{ker} \omega$ is essential in $\mathcal{B}\}$ is a two sided ideal of $S$.

Proof: Since the zero homomorphism in $S$ and its kernel is $\mathcal{B} \leq_{e} \mathcal{B}$, we have $W(S) \neq \emptyset$. Now let $\alpha, \beta \in W(S$,$) and f \in S$, then $\operatorname{ker} \alpha \leq_{e} \mathcal{B}$ and $\operatorname{ker} \beta \leq_{e} \mathcal{B}$, since $\operatorname{ker} \alpha \cap \operatorname{ker} \beta \leq \operatorname{ker}(\alpha+\beta)$, then $\operatorname{ker}(\alpha+\beta)$ essential subsemimodule of $\mathcal{B}$, so $\alpha+\beta \in W(S)$. Also ker $f \alpha=\alpha^{-1}($ ker $f) \supseteq$ $\operatorname{ker} \alpha$ (since $\operatorname{ker} \alpha$ is essential, then $\left.\operatorname{ker} f \alpha \leq_{e} \mathcal{B}\right)$. Finally ker $\alpha f=f^{-1}(\operatorname{ker} \alpha)$ by Lemma (1) ker $\alpha f$ $\leq_{e} \mathcal{B}$. Hence $f \alpha, \alpha f \in W(S)$. IIII

Note: Let $A_{\omega \beta}=\{m \in \mathcal{B} \mid m=\beta(\omega(m))\}$, where $\omega, \beta \in S$.

Lemma 3. If $\mathcal{B}$ is a P.Q.- injective semimodule and $\omega \in S$, then $\operatorname{ker} \omega$ is essential in $\mathcal{B}$ iff $A_{\omega \beta}=0$ for all $\beta \in S$.

Proof: Assume that $A_{\omega \beta}=0 \quad \forall \beta \in S$ and ker $\omega \cap \mathcal{R} b=0, \quad b \in \mathcal{B}, \quad$ then $\quad l_{\mathcal{R}}\left(\omega(\mathrm{b}) \subseteq l_{\mathcal{R}}(b)\right.$ by Proposition(3. 17)(1) it follows $b \in S, \omega($ b) this means $b=\beta(\omega(b))$ for some $\beta \in S$ so $b=0$, then $\operatorname{ker} \omega \leq_{e}$ B.i.e., $\omega \in W(S)$.

Now, assume $A_{\omega \beta} \neq 0$ for some $\beta \epsilon S$, that is $\exists 0 \neq b \in \mathcal{B}$ such that $b=\beta(\omega(b))$, then $\left.\beta \omega\right|_{\mathcal{R} b}=$ $1_{\mathcal{R} b} \quad$ which implies that $\operatorname{ker} \beta \omega \cap \mathcal{R} b=0$, then $\operatorname{ker} \omega \cap \mathcal{R} b=0(\operatorname{ker} \omega \leq \operatorname{ker} \beta \omega)$. Therefor $\omega \notin$ $W(S) . \quad / I /$,

Note: By the above Lemma $W(S)=\left\{\omega \in S \mid A_{\omega \beta}=\right.$ 0 for all $\beta \in S$, \}. 
Lemma 4. Let $I$ be a subtractive ideal of the subtractive semiring $\mathcal{R}$, then $I \subseteq \operatorname{Rad}(\mathcal{R})$ if and only if each element of the coset $1+l$ has an inverse.

Proof: $(\Rightarrow)$ Assume that $1+a$ is not invertible for some $a \in I$, then $1+a$ must belongs to some maximal ideal $J$ of $\mathcal{R}$, now $a \in \operatorname{Rad}(\mathcal{R})$ implies $a \in$ $J$ and $1+a \in J$ (since $J$ is subtractive), then $1 \in J$ and so $J=\mathcal{R}$ this contradicts the maximality of $J$. Therefor $1+a$ is invertible.

$\Leftarrow$ If each member of $1+I$ has an inverse in $\mathcal{R}$, but $I$ is not contained in $\operatorname{Rad}(\mathcal{R})$ that is, there is a maximal ideal $J$ of $\mathcal{R}$ not containing $I$. If $a \in I$ and $a \notin J$ then $(J, a)=\mathcal{R} \Rightarrow 1=u+r a$ for some $u$ in $J$ and $r$ in $R$. we have $1+0=u+r a(0, r a \in l) \Rightarrow 1+I=u+!$ $\Rightarrow u \in 1+I \Rightarrow u$ is invertible, a contradiction,$\Rightarrow$ $I \subseteq \operatorname{Rad}(\mathcal{R})$. III/

Lemma 5. For any element $a$ of $\mathcal{R}, a \in \operatorname{Rad}(\mathcal{R})$ if and only if $1+r a$ is invertible for all $r \in \mathcal{R}$.

Proof: If $a \in \operatorname{Rad}(\mathcal{R})$, then $(a) \subseteq \operatorname{Rad}(\mathcal{R})$ by Lemma(4) we have, each element of the coset $1+(a)$ is invertible $\Rightarrow 1+r a$ has inverse where $r \in \mathcal{R}$. Conversely, let $1+r a$ is invertible, if $a \notin \operatorname{Rad}(\mathcal{R})$, then for some maximal ideal $\hat{\jmath}, a \notin J$ which implies $\mathcal{R} \mathrm{a}+\hat{\jmath}=\mathcal{R} \Rightarrow r a+j=1$ for some $j$ in $\hat{\jmath}$, that is, $r a+j$ $=0+1$ implies that, $j \in 1+(a)$ hence $j$ is invertible and this is a contradiction. //I/

The following is illustration of some relationships between $\operatorname{Rad}(S)$ and concepts $W(S)$ and $Z(S$,$) on$ P.Q.-injective semimodule. Where those properties are mentioned in (6) and (12) for modules.

Proposition 6. Let $\mathcal{B}$ be P.Q.-injective semimodule with $S$.

(a) $Z(S, \subseteq) \subseteq W(S$,

(b) If $\mathcal{B}$ is uniform, then $Z(S,) \subseteq \operatorname{Rad}(S)$

(c) If every monomorphism in $S$, has right inverse then $W(S, \subseteq \operatorname{Rad}(S)$.

Proof: (a) Let $\alpha \notin W(S)$, then ker $\alpha$ is not essential in $\mathcal{B} \Rightarrow \operatorname{ker} \alpha \cap \mathcal{R} b=0$ where $0 \neq b \in \mathcal{B}$ hence $\left.\alpha\right|_{\mathcal{R} b}: \mathcal{R} b \rightarrow \mathcal{B}$ is monic, since $\mathcal{B}$ is P.Q.-injective, then there is $\beta: \mathcal{B} \rightarrow \mathcal{B}$ such that $\alpha \beta=$ $1_{\mathcal{B}}$ implies $\alpha \beta(b)=b \Rightarrow \alpha \notin \mathcal{Z}(S)$ ( if $t \in l(\alpha$ )$\Rightarrow t \alpha=0,(t \alpha) \beta=0 \Rightarrow t(\alpha \beta)=0 \Rightarrow t=0$, so $l(\alpha)=0$ but this a contradiction.

(b) If $\alpha \in Z(S) \Rightarrow l(\alpha)$ is essential ideal of $S \Rightarrow$ $\operatorname{ker}(\alpha) \neq 0$ ( let $\operatorname{ker}(\alpha)=0$, if $t \in l(\alpha) \Rightarrow t \alpha=0$, then $\exists f$ such that $\alpha f=1 \Longrightarrow(t \alpha) f=0=t(\alpha f) \Rightarrow t=0$ this contradiction with essential). For any $\beta \in S$ we have $\operatorname{ker}(\alpha) \cap \operatorname{ker}(1+\beta \alpha)=0$ (since $\mathcal{B}$ is uniform) we have $\operatorname{ker}(1+\beta \alpha)=0 \Longrightarrow(1+\beta \alpha) f=1_{\mathcal{B}} \Rightarrow \alpha \in$ $\operatorname{Rad}(S)$.

(c) If $\alpha \in W(S) \Rightarrow \operatorname{ker}(\alpha) \leq_{e} \mathcal{B}$, ker $(\alpha) \cap$ $\operatorname{ker}(1+\alpha)=0$ we get $\operatorname{ker}(1+\alpha)=0$, hence $1+\alpha$ has right inverse by hypotheses, then $\alpha \in \operatorname{Rad}(S)$. IIII
In (6) the notion kasch module was introduced, similarly, we introduce this concept for semimodule as follows:

Definition 7. (3). An $\mathcal{R}$-semimodule $\mathcal{B}$ is called a kasch semimodule if every simple sub quotient $A$ of $\mathcal{B}$ is embedded in $\mathcal{B}$. i.e., there is monomorphism from $A$ into $\mathcal{B}$.

Example 8. let $\mathcal{B}=\mathbb{Z}_{6}=2 \mathbb{Z}_{6} \oplus 3 \mathbb{Z}_{6}$. Since $\mathbb{Z}_{6} / 2 \mathbb{Z}_{6} \cong 3 \mathbb{Z}_{6}$, then $3 \mathbb{Z}_{6}$ embeds in $\mathbb{Z}_{6}$, i.e. there is monomorphism $\alpha: 3 \mathbb{Z}_{6} \rightarrow \mathbb{Z}_{6}$, similarly $\mathbb{Z}_{6} / 3 \mathbb{Z}_{6}$, but $\mathbb{Z}$ over itself is not kasch semimodule

Lemma 9. (3). Let $\mathcal{B}$ be a P.Q.-injective semimodule which is kasch semimodule. if $I$ is maximal ideal of $\mathcal{R}$, then $r(I) \neq 0$ if and only if $l(m) \subseteq I$ for some $0 \neq m \in \mathcal{B}$. Particularly, $r(I)$ is a simple as right $S$-simimodule. Where $r(I)=\{b \in \mathcal{B}$ $\mid u b=0, \forall u \in I\}$ and $l(m)=\{t \in \mathcal{R} \mid t m=0\}$.

Proposition 10. If $\mathcal{B}$ is a P.Q.-injective which is kasch semimodule, then $W(S)=r_{S}(\operatorname{Soc}(\mathcal{B}))$.

Proof: Since $\operatorname{Soc}(\mathcal{B}) \leq_{e} \mathcal{B}$ by Proposition(3.31.) (3). Let $\alpha \in W(S) \Rightarrow \operatorname{ker}(\alpha) \leq_{e} \mathcal{B}$, then $\operatorname{Soc}(\mathcal{B}) \subseteq$ $\operatorname{ker}(\alpha) \Rightarrow \operatorname{Soc}(\mathcal{B}) \alpha=0 \Rightarrow \alpha \in r_{\mathrm{S}}(\operatorname{Soc}(\mathcal{B}))$. On the other hand, $\operatorname{Soc}(\mathcal{B}) \alpha=0$ implies $\operatorname{ker}(\alpha) \leq_{e} \mathcal{B}$ and $\alpha \in W(S)$. III/

Proposition 11. Let $\mathcal{B}$ be a P.Q.-injective semimodule which is kasch semimodule with $S$, then $\operatorname{Soc}\left({ }_{R} \mathcal{B}\right)=\operatorname{Soc}\left(\mathcal{B}_{S}\right) \subseteq r(\operatorname{Rad}(\mathcal{R}))$

Proof: $\operatorname{Soc}\left({ }_{R} \mathcal{B}\right)=\operatorname{Soc}\left(\mathcal{B}_{S}\right)$ from (3). Let $b \in$ $\operatorname{Soc}\left(\mathcal{B}_{S}\right)$. If $\mathcal{R} b$ is a simple in ${ }_{R} \mathcal{B}$, then $b S$ is a simple in $\mathcal{B}_{\text {s. }}$. Let $I$ be a maximal ideal in $\mathcal{R}, ! b=$ $\mathcal{R} b$ or $l b=0$. If $l b=\mathcal{R} b$ there exists $i \in I$ such that $b=i b$ implies $i=1$, but this contradiction, then $I b=0$ and hence $b \in r(I)) \subseteq r(\operatorname{Rad}(\mathcal{R}))$. III/

Lemma 12. For $b$ in a local semiring $\mathcal{R}$, then $\mathcal{R} b$ is small in $\mathcal{R}$ if and only if $b \in \operatorname{Rad}(\mathcal{R})$.

Proof: Let $\mathcal{R} b$ be small in $\mathcal{R}$ implies, $b \in \mathcal{R} b, \mathcal{R} \neq$ $\mathcal{R} b$ then $b$ is not invertible, hence $b \in \operatorname{Rad}(\mathcal{R})$. Conversely, suppose that $b \in \operatorname{Rad}(\mathcal{R})$ and $l$ is an ideal of $\mathcal{R}$ with $I+\mathcal{R} b=\mathcal{R}$. If $I \neq \mathcal{R}$ then, by Zorn's Lemma there is a maximal ideal $J$ of $\mathcal{R}$ with $b \notin \mathrm{J}$. Since $J+\mathcal{R} b=\mathcal{R}$ and $b \in \operatorname{Rad}(\mathcal{R}) \subset J$, a contradiction hence $I=\mathcal{R}$, consequently $\mathcal{R} b$ is small. I/I/

In (13) the notion "P-injective ring" was introduced, where the ring $\mathcal{R}$ is said to be $\mathrm{P}$ injective if ${ }_{R} \mathcal{R}$ is a P.Q.-injective module. Analogous, we introduce this notion for semiring as follows, a semiring $\mathcal{R}$ is called $\mathrm{P}$-injective if ${ }_{R} \mathcal{R}$ is a P.Q.- injective semimodule.

In (13) the next result was proved for ring. We prove this result for semiring, but in different way (we will add the conditions $r(b)+a \mathcal{R}$ is subtractive subsemimodule of ${ }_{R} \mathcal{R}$ and ${ }_{R} \mathcal{R}$ is semisubtractive semimodule) as follows. 
Proposition 13. If $\mathcal{R}$ is left $\mathrm{P}$-injective semiring, then $\operatorname{Rad}(\mathcal{R})=\mathcal{Z}\left({ }_{R} \mathcal{R}\right)$. Provided that $r(b)+a \mathcal{R}$ is subtractive subsemimodule of ${ }_{R} \mathcal{R}$ and ${ }_{R} \mathcal{R}$ is semisubtractive semimodules

Proof: If $a \in Z\left({ }_{R} \mathcal{R}\right)$, then $l(a) \leq_{e} \mathcal{R}$. But, $l(a) \cap l(1+a)=0)[s a=0$ and $s(1+a)=0 \Longrightarrow s=0]$, so, $l(1+a)=0$. Hence $r[l(1+a)]=\mathcal{R}$ which implies $\mathcal{R}=(1+a) \mathcal{R}$ by Proposition (3.20.(iii))(3), which means $1+a$ is invertible and $a \in \operatorname{Rad}(\mathcal{R})$, that is $\mathcal{Z}\left({ }_{R} \mathcal{R}\right) \subseteq \operatorname{Rad}(\mathcal{R})$. Conversely, if $a \in$ $\operatorname{Rad}(\mathcal{R})$ and $l(a) \cap \mathcal{R} b=0, \quad b \in \mathcal{R}, \quad$ by Proposition(3. 21.) (iv) ) (3), $r[\mathcal{R} b \cap l(a)]=r(b)+a \mathcal{R}$, that is, $r(b)+a \mathcal{R}=\mathcal{R}$, but $a \mathcal{R}$ is small since $a \in$ $\operatorname{Rad}(\mathcal{R})$ by Lemma(12.), so $r(b)=\mathcal{R}$ which implies $b=0$, and hence $l(a) \leq_{e} \mathcal{R}$, therefore $a \in \mathcal{Z}(\mathcal{R})$. IIII

In(6) the following condition was added to module which is related to P.Q.-injective, similarly, we introduce this condition for semimodule.

Definition 14. A semimodule $\mathcal{B}$ has the $\mathrm{C}_{2^{-}}$ condition, if every subsemimodule of $\mathcal{B}$ that is isomorphic to a direct summand of $\mathcal{B}$ is itself a direct summand of $\mathcal{B}$.

Lemma 15.(3) Let $\mathcal{B}$ be a subtractive, semisubtractive and cancellative $\mathcal{R}$ - semimodule, then $\mathcal{B}=U \oplus C$ if and only if $\mathcal{B}=U+C$ and $U \cap$ $C=\{0\}$.

In literatures, the notion of split monomorphism or epimorphism were not found, only split exact sequences where defined, see (14). In the following we will give suitable conditions to define split monomorphism of semimodule.

Lemma 16. If $\mathcal{B}$ is subtractive, semisubtractive and cancellative semimodule, then a subsemimodule $U$ of $\mathcal{B}$ is a direct summand of $\mathcal{B}$ if and only if the inclusion map $i: U \rightarrow \mathcal{B}$ has left inverse.

Proof: $(\Longrightarrow)$ is clear.

$(\Longleftarrow)$ Assume that $\alpha: \mathcal{B} \rightarrow U$ is a homomorphism such that $\alpha i=1_{\mathrm{U}}$. Let $C$ $=\operatorname{ker} \alpha$, then $u \in U \cap C ̧$ implies

$\alpha(u)=u$ and $\alpha(u)=0 \Rightarrow u=0$, that is $U \cap C ̧=0$.

Claim. $\mathcal{B}=U \oplus C$, we must to show that $\mathcal{B}=U$

$+C$. Let $b \in \mathcal{B}$, then $\alpha(b) \in U$. B is semisubtractive implies either $b=\alpha(b)+h$ or $b+k=\alpha(b)$ for some $h, k \in \mathcal{B}$

Case1 $b=\alpha(b)+h \Rightarrow \alpha(b)=\alpha(\alpha(b))+\alpha(h) \Rightarrow$ $\alpha(b)=\alpha(b)+\alpha(h) \Rightarrow \alpha(h)=0 \Rightarrow h \in C \Longrightarrow b \in U$ $+C[\alpha(\alpha(b))=\alpha(i(\alpha(b))=\alpha i(\alpha(b))=\alpha(b)]$

Case $2 b+k=\alpha(b)$, also implies $k \in C$. Now $k \in$ $C ̧ \Rightarrow k \in U \oplus C, \alpha(b) \in U \Longrightarrow \alpha(b) \in U \oplus C$. But $U$ $\oplus C$ is a subtractive subsemimodule of $\mathcal{B}$, so we get $b \in U \oplus C$. Therefor $\mathcal{B}=U \oplus C$, which means $U$ is a direct summand of $\mathcal{B}$. I/I/

Remark 17. If $\mathcal{B}$ is subtractive, semisubtractive and cancellative semimodule, $\alpha: U \rightarrow \mathcal{B}$ is said to split monomorphism if there exists a homomorphism $\gamma: \mathcal{B} \rightarrow U$ such that $\gamma \alpha=1_{\mathrm{U}}$.

Proposition 18. Every quasi-injective semimodule has $\mathrm{C}_{2}$-condition.

Proof: Let $U$ be a direct summand of $\mathcal{B}$ and $\vartheta$ : $U^{\prime} \rightarrow U$ be an isomorphism, $\pi: \mathcal{B} \rightarrow U$ be the projection map. If $\phi: \mathcal{B} \rightarrow \mathcal{B}$ is an extension of $\vartheta$, such that $\phi i^{\prime}=i \quad \vartheta$, then $\left(\vartheta^{-1} \pi\right) \phi i^{\prime}=\left(\vartheta^{-1} \pi\right) i \vartheta$ $=\left(\vartheta^{-1} \pi \phi\right) i^{\prime}=\vartheta^{-1}(\pi i) \vartheta=\vartheta^{-1} 1_{\mathrm{U}} \vartheta=\vartheta^{-1} \vartheta=1_{\mathrm{U}}$, then $i^{\prime}$ has left inverse and hence $U^{\prime}$ is a direct summand of $\mathcal{B}$ by Lemma(16).

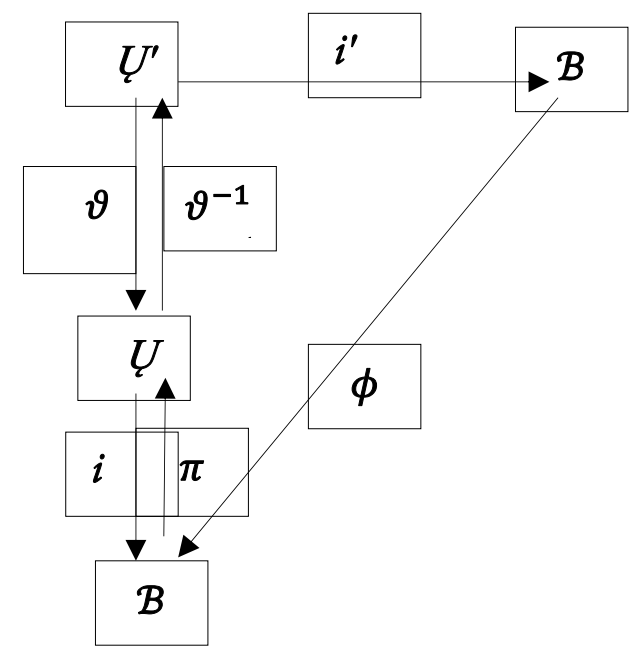

\section{IIII}

Lemma 19. If $\mathcal{B}$ is subtractive, semisubtractive and cancellative semimodule with S. If $\mathcal{B}$ has the $\mathrm{C}_{2^{-}}$ condition, then $W(S) \subseteq \operatorname{Rad}(S)$.

Proof: $\operatorname{ker}(\theta) \cap \operatorname{ker}(1+\theta)=0$ always holds. If ker $\theta$ is essential in $\mathcal{B}$, then $\operatorname{ker}(1+\theta)=0$ and hence $1+\theta$ is monic. $\operatorname{Im}(1+\theta) \cong \mathcal{B}$ let $g: \operatorname{Im}(1+\theta$ )$\rightarrow \mathcal{B}$ be the isomorphism $b(1+\theta) \mapsto b$. Since $\mathcal{B}$ has the $C_{2}$-condition, then $\operatorname{Im}(1+\theta)$ is a direct summand of $\mathcal{B}(\mathcal{B}$ is a direct summand of $\mathcal{B})$ this means $\mathcal{B}=\operatorname{Im}(1+\theta) \oplus \mathcal{B}^{\prime}$ for some $\mathcal{B}^{\prime} \leq \mathcal{B}$, let $\alpha \in S$ defined by $\alpha(b)=g(b)$ if $b \in \operatorname{Im}(1+\theta), \alpha$ (b) $=0$ if $b \in \mathcal{B}^{\prime}$, then $\alpha$ is well defined and $\alpha(1+\theta$ )$=1_{\mathcal{B}}$ and hence $\theta \in \operatorname{Rad}(S)$. IIII

Remark 20. If $\mathcal{B}$ is subtractive, semisubtractive and cancellative quasi-injective semimodule, then $W(S)$ $\subseteq \operatorname{Rad}(S)$.

Proof: Since $\mathcal{B}$ has $\mathrm{C}_{2}$-condition by Proposition(18) and by Lemma(19), we have $W(S) \subseteq \operatorname{Rad}(S$,$) . II/I$

The following characterization explain the relationship between the $\mathrm{C}_{2}$-condition and cyclic P.Q.-injective semimodule. 
Proposition 21. Let $\mathcal{B}$ be a P.Q.-injective semimodule with S.

(a) If $U$ and $U^{\prime}$ are isomorphic cyclic subsemimodules of $\mathcal{B}$ and $U^{\prime}$ is a direct summand, then $U$ is also a direct summand of $\mathcal{B}$.

(b) Every cyclic P.Q.-injective semimodule satisfies $\mathrm{C}_{2}$-condition.

Proof: Since a direct summand of a cyclic semimodules is cyclic, it is enough to prove (a), similar to Proposition (18) with take $U=\mathcal{R} u$ and $U^{\prime}$ $=\mathcal{R} u^{\prime}$.

(b) From (a). I/I/

Proposition 22. If $\mathcal{B}$ is a cyclic, subtractive, semisubtractive and cancellative semimodule P.Q.injective semimodule with $S$, then $Z(S) \subseteq \operatorname{Rad}(S)$.

Proof:

Since $\mathcal{B}$ is P.Q.-injective, then by Proposition (6 (b)) $Z(S, \subseteq W(S$, ). Moreover by Proposition $(22) \mathcal{B}$ has $\mathrm{C}_{2}$-condition, therefore $W(S) \subseteq \operatorname{Rad}(S)$ (by Lemma(19) $W(S,) \subseteq \operatorname{Rad}(S)$. We have $\mathcal{Z}(S) \subseteq$ $\operatorname{Rad}($ S)..$/ / / /$

In (6) principally self-generator module was given. In the following we given an analogous of that notion for semimodule and related relation.

Definition 23. (3). An $\mathcal{R}$-semimodule $\mathcal{B}$ is said to be principally self-generator if for every element $b \in \mathcal{B}$, there exists an epimorphism $\alpha: \mathcal{B} \rightarrow \mathcal{R} b$, and then there exists $b^{\prime} \in \mathcal{B}$ such that $\alpha\left(b^{\prime}\right)=b$.

Proposition 24. Let $\mathcal{B}$ be a principally self generator semimodule with Ș. Then $Z(S, S)=W(S)$ ).

Proof: Let $\omega \in W(S)$. Take $0 \neq \alpha \in S$ we have ker $\omega \cap \alpha(\mathcal{B}) \neq 0$, say $0 \neq \alpha(b) \in \operatorname{ker} \omega$.

Since $\mathcal{B}$ is principally self - generator, then $b=\lambda\left(b_{1}\right)$ where $\lambda: \mathcal{B} \rightarrow \mathcal{R} b$, then $\alpha \lambda \neq 0$, since $\alpha \lambda\left(b_{1}\right)=$ $\alpha(b) \neq 0$, but $\omega \alpha \lambda=0$ because $\omega \alpha \lambda(\mathcal{B}) \subseteq \omega \alpha(\mathcal{R} b)$ $=\mathcal{R} \omega \alpha(b)=0$. Hence $0 \neq \alpha \lambda \in r(\omega) \cap S, \lambda$. This means $\omega \in \mathcal{Z}(S)$. Conversely, if $\omega \in \mathcal{Z}(S)$ and $0 \neq$ $b \in \mathcal{B}$, we must show that $\operatorname{ker} \omega \cap \mathcal{R} b \neq 0$. Since $\mathcal{B}$ is principally self -generator, then there is epimorphism $\lambda: \mathcal{B} \rightarrow \mathcal{R} b$ and $\lambda \neq 0$, so $r(\omega) \cap$ $S, \lambda \neq 0$, let $\omega \alpha \lambda=0$ then $\alpha \lambda \in r(\omega) \cap S, \lambda$ for some $\alpha \in S$ where $\alpha \lambda \neq 0$. Let $\alpha \lambda\left(b_{1}\right) \neq 0, b_{1} \in \mathcal{B}$, we have $\alpha \lambda\left(b_{1}\right) \in \alpha(\mathcal{B})=\mathcal{R} b$, so write $\alpha \lambda\left(b_{1}\right)=t b$ where $\mathrm{t} \in \mathcal{R}$, then $\omega(t b)=\omega\left[\alpha \lambda\left(b_{1}\right)\right]=0$, so $0 \neq t b \in$ ker $\omega \cap \mathcal{R} b$, then $\omega \in W(S)$. IIII

\section{Conclusion:}

Some results are obtained in this paper, some conditions and Lemma of semimodules have been added to get similar results and characteristics of modules. The Jacobson radical of endomorphism rings has been expended for endomorphism semirings of a P.Q.-injective semimodule, also some properties of this notion are discussed.

\section{Authors' declaration:}

- Conflicts of Interest: None.

- Ethical Clearance: The project was approved by the local ethical committee in University of Babylon.

\section{References:}

1. Golan JS. Semirings and Their Applications, Kluwer Academic Publishers, Dordrecht, the Netherlands, 1999.

2. Kasch F. Modules and Rings. Academic press, London, New York, 1982.

3. Alhossaini AM, Aljebory KS. Principally QuasiInjective Semimodules. Baghdad Sci. J., 2019;16(4):928-936.

4. Gupta V, Chaudhari JN. Right Local Semiring, EJM, 2013; 6(1), 5 pages.

5. Alhossaini AM. Alsaebari SH. On Periradical of Semimodules. Baghdad Sci. J. 2018; 15(4),472-478.

6. Nicholoson WK, Park JK, Yousif MF. Principally Quasi-Injective Modules. COMMUN ALGEBRA.1999; 27(4):1683 -1693.

7. Alhossaini AM, Aljebory ZA. On p-duo Semimodules. J. of Univ. of Babylon, Pure and Appl. Sci. 2018; 26(4).

8. Nazari RR, Ghalandazaden S. Content Semimodules. Exta. Math. 2017; 32( 2): 239-254.

9. Diop E ,Sow D. On Essential Subsemimodules and Weakly Co-Hopfion Semimodules. Eur. J. of Pure Appl. Math. 2016; 9(3): 250-265.

10. Abdulameer, H. Fully stable semimodules, master degree thesis, mathematics, Babylon University, 2017.

11. bdulameer H, Husain AM. Fully stable semimodules. Albahir J.2017; 5(9) and (10):13-20.

12. Wongwal S. On The Endomorphism Ring of a Semiinjective Module. Acta. Math. Univ. Comenianae. (2002); 1, 27-33.

13. Nicholoson WK, Yousif MF. Principally Injective Rings. J. Algebra. 1995; (174): 77-93.

14. Chaudhari JN, Bonde DR. On Exact Sequence of Semimodules over Semirings. ISRN Algebra. 2013;1- 
جذر جاكوبسون لثبه حلقة التثاكلات في شبه المقاسات الرئيسية شبه الاغماريه

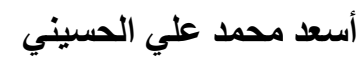

\section{ختام صاحب حمزه الجبوري}

قسم الرياضيات، كلية التربية للعلوم الصرفة، جامعة بابل، بابل، العراق.

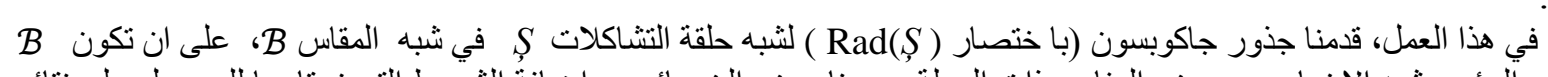

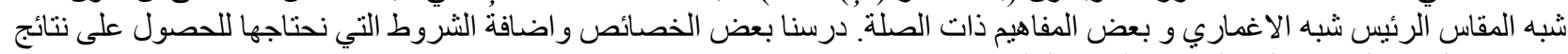

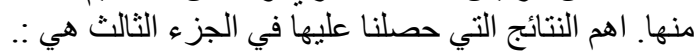

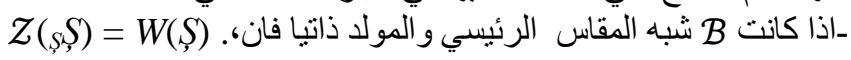

الكلمات المفتاحية: جذر جاكوبسون لشبه الحلقة، شبه الحلقة، شبه المقاس، شبه المقاس الرئيس، شبه المقاس الرئيس شبه الاغماري. 